\title{
Enhancing light scattering effect of white LEDs with ZnO nanostructures
}

\author{
My Hanh Nguyen Thi ${ }^{1}$, Nguyen Thi Phuong Loan ${ }^{2}$, Hoang Van Ngoc $^{3}$ \\ ${ }^{1}$ Faculty of Mechanical Engineering, Industrial University of Ho Chi Minh City, Vietnam \\ ${ }^{2}$ Faculty of Fundamental 2, Posts and Telecommunications Institute of Technology, Vietnam \\ ${ }^{3}$ Institute of Applied Technology, Thu Dau Mot University, Vietnam
}

\begin{tabular}{l} 
Article Info \\
\hline Article history: \\
Received May 17, 2020 \\
Revised Apr 8, 2021 \\
Accepted Apr 18, 202 \\
\hline Keywords: \\
$\mathrm{CaCO}_{3}$ \\
$\mathrm{CaF}_{2}$ \\
$\mathrm{Color}$ \\
$\mathrm{Luminous}$ unity flux \\
$\mathrm{Mie}^{-}$scattering theory \\
$\mathrm{SiO}_{2}$ \\
$\mathrm{TiO}_{2}$
\end{tabular}

Corresponding Author:

Hoang Van Ngoc

Institute of Applied Technology

Thu Dau Mot University

No 6, Tran Van On Street, Thu Dau Mot city, Binh Duong province, Vietnam

Email: ngochv@tdmu.edu.vn

\begin{abstract}
Pc-LEDs, the lighting method that blends blue LED light with yellow light from phosphor to discharge white radiation, is one of the most advance known for high lumen output. However, pc-LEDs has inferior due to angular CCT deviation, which prevent pc-LEDs from reaching better performance. As a result, this research is conducted to address the need of pc-LEDs development by introducing a configuration doped with $\mathrm{ZnO}$ nanoparticles. The finite-difference time-domain (FDTD) method and the phosphor layer containing $\mathrm{ZnO}$ were applied in the experiments. The effect of $\mathrm{ZnO}$-filled on the performance of color quality pc-LEDs is confirmed through calculated results. In particular, the uniformity of scattered light is improved with the presence of $\mathrm{ZnO}$. In addition, $\mathrm{ZnO}$ particles also minimize the deviation of color temperature and enhance the color quality. Although there is a small decline in lumen output to achieve better color temperature uniformity, however, with suitable concentrations such as $0.25 \% \mathrm{~N}-\mathrm{ZnO}, 0.25 \% \mathrm{~S}-\mathrm{ZnO}$, and $0.75 \% \mathrm{R}-\mathrm{ZnO}$, the decline is acceptable. The research on $\mathrm{ZnO}$ pc-LEDs demonstrates that this affordable and simple configuration can improve lighting properties and create other directions to enhance white light.
\end{abstract}

This is an open access article under the CC BY-SA license.

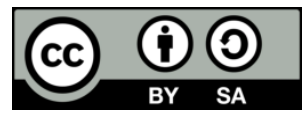

\section{INTRODUCTION}

The among many approaches in fabricating white light, the configuration that converts light from phosphor (pc-LEDs) is currently the most proficiency and common. Comparing to previous light sources, pc-LEDs is better in both amount and quality of light while using less energy, less manufacturing expense, and does not emit chemical waste [1]-[4]. Blue LED and yttrium aluminum garnet (YAG:Ce) phosphor are the ingredients in the mixing process to create white light in pc-LEDs [5], [6]. Despite being widely use, this quality of light fabricated from this method is bad due to the ingredients being unevenly distributed causing the color temperature to deviate. Deviated color temperature hinder the color quality by inducing a yellow ring at the edge of discharged light that can only be addressed with structural reformation [7]-[9]. Although the optical qualities can be improved by using modified configurations suggested from research such as conformal coating structure [10], phosphor material adjustment [11], changing discharge surface [12], and structural modification [13], [14]. These suggestions are all capable of improving the color quality, however, complexity and high production cost prevents them from being widely used. Therefore, an alternative 
solution with simple production process, low manufacturing expense while obtaining similar effects on optical properties is the target for researchers.

Currently, the attention is on scattering enhancement materials that is cost-effective and can be easily integrated into the lighting structure. Among the enhancement materials, $\mathrm{TiO} 2, \mathrm{ZrO} 2$ and $\mathrm{SiO} 2$, the metal oxide compounds, are proven as effective materials in promoting angular CCT uniformity, a results concluded from experiments [15]-[18]. The $\mathrm{ZrO}_{2}$ with $300 \mathrm{~nm}$ of particle size can reduce color temperature deviation by $580 \mathrm{~K}$ from $1000 \mathrm{~K}$ to $420 \mathrm{~K}$ in between $-70^{\circ}$ to $70^{\circ}$ angles, a result obtained the research conducted by Chen et al. [15]. Another scattering materials, $\mathrm{TiO}_{2}$, is proved as effective in limiting CCT diversion through experiments by Lee et al. [18]. The $\mathrm{TiO}_{2}$ particles are mixed into the phosphor material and as the concentration of $\mathrm{TiO}_{2}$ increases the color temperature deviation declines. These research provide valuable information on light quality control, however, it is not enough for the development demands considering how only the concentrations are changed while the particles modifications are completely disregarded [5], [19], [20]. Without an extensive research that cover all aspects of diffuser utilization and provide a detailed instruction, diffuser particle selection is still a complex matter.

In this research study the $\mathrm{ZnO}$ particle as an diffuser to enhance scattering effect of pc-LED because this metal oxide diffuser is simple, cheap and has tunable particle structure [21]. The key factor to perform experiments on $\mathrm{ZnO}$ particles is control over thermal performance, timing, and particles density. The lighting device react differently with each change of the mentioned factors and that affects optical properties. $\mathrm{ZnO}$ with refractive index of 2 can fit between 2.5 index of $\mathrm{GaN}$ and 1.0 of air serving as a gradient refractive index layer. The usage of $\mathrm{ZnO}$ nanoparticles in lighting device with $\mathrm{GaN}$ semiconductor has been reported to enhance lumen output [22]. Yin et al. used $\mathrm{ZnO}$ particles with two distinct patterns engraved on particle surface, micro-cylinders and nanorods array, also achieved higher light output [23]. In another research, Yin et al. presented a different high scattering enhancement configuration by applying $\mathrm{ZnO}$ particles with uneven surface on a flat ITO layer [24]. Lee et al. experiment results also suggested that $\mathrm{ZnO}$ with modified surface is effective in managing lighting efficiency of GaN LEDs [25]. The many techniques to utilize ZnO particles as light transmission enhancer were introduced by previous studies, however, diffusing the $\mathrm{ZnO}$ on the lighting structure to achieve better optical properties was rarely mentioned. The effects of $\mathrm{ZnO}$ surface structure on a few optical properties such as correlated color temperature and color quality of pc-LEDs were lacking, which makes choosing an optimal method to enhance lighting performance difficult. Therefore, this research aims to fulfill those criteria by using different-shape $\mathrm{ZnO}$ from node-like $(\mathrm{N}-\mathrm{ZnO})$, sheet-like ( $\mathrm{S}$ $\mathrm{ZnO})$, to rod-like $(\mathrm{R}-\mathrm{ZnO})$ on pc-LEDs and study their results. The experiments on $\mathrm{ZnO}$ doped lighting configurations are executed with the support of scattering energy distribution calculation and finite-difference time-domain (FDTD) methods. $\mathrm{ZnO}$ proficiency in enhancing scattering event and color uniformity were approved by the experimental results. In another finding, forming an encapsulation of $\mathrm{ZnO}$ particles with different shape and amount joined by silicone gel can significantly boost CCT uniformity of the lighting configuration.

\section{RESEARCH METHOD}

According to Mie-scattering theory, when the $\mathrm{ZnO}$ particles in conformal phosphor pc-LEDs structure induce light scattering, at that time the effect will be determined by apply a calculating tool called MATLAB [22]-[25]. The equations applied to calculate the scattering coefficient $\mu$ sca $(\lambda)$, the anisotropy factor $\mathrm{g}(\lambda)$, the reduced scattering coefficient $\delta$ sca $(\lambda)$ and the scattering amplitude functions $S_{1}(\theta)$ and $S_{2}(\theta)$ are presented as:

$$
\begin{aligned}
& \mu_{s c a}(\lambda)=\int N(r) C_{s c a}(\lambda, r) d r \\
& g(\lambda)=2 \pi \iint_{-1}^{1} p(\theta, \lambda, r) f(r) \cos \theta d \cos \theta d r \\
& \delta_{s c a}=\mu_{s c a}(1-g) \\
& S_{1}=\sum_{n=1}^{\infty} \frac{2 n+1}{n(n+1)}\left[a_{n}(x, m) \pi_{n}(\cos \theta)+b_{n}(x, m) \tau_{n}(\cos \theta)\right] \\
& S_{2}=\sum_{n=1}^{\infty} \frac{2 n+1}{n(n+1)}\left[a_{n}(x, m) \tau_{n}(\cos \theta)+b_{n}(x, m) \pi_{n}(\cos \theta)\right]
\end{aligned}
$$

Where, $N(r)$ represents the amount of scattering particles (per $\mathrm{mm}^{3}$ ). $C_{s c a}$ is the scattering cross section $\left(\mathrm{mm}^{2}\right)$. The wavelength of incident light is expressed by $\lambda(\mathrm{nm})$. The parameter of particles size is $r$ for radius 
$(\mathrm{mm})$. The degree in which the scattering event occurs is depicted by $\theta$. the phase function is shown as $p(\theta, \lambda, r)$ while the function for diffusor size distribution in phosphor film is $f(r)$. The expansion coefficient for even and odd symmetry are respectively as $a_{n}$ and $b_{n} . x$ is the size parameter, $m$ is index of refraction. $\pi_{n}(\cos \theta)$ and $\tau_{n}(\cos \theta)$ are the functions for dependence in angular.

With minimal amount such as $0.25 \%$, the $\mathrm{ZnO}$ particles application does not induce much discrepancy in scattering light distribution among different $\mathrm{ZnO}$ structure types as shown in Figures 1 and 2. This is due to the lack of $\mathrm{ZnO}$ particles at low particle density, making it harder for $\mathrm{ZnO}$ to impact the scattering event, thus, the influence of $\mathrm{ZnO}$ structure on the lighting performance is quite subtle at this point. As the diffuser particles concentration being too low to prompt any changes, the total scattered light according to common scattering theory is solely based on particle individual scattering. However, the difference between pc-LEDs with and without $\mathrm{ZnO}$ are still visible. The influences of $\mathrm{ZnO}$ begin to show as the concentration thickens.

According to the results shown in Figures 3 and 4, it can be concluded that $\mathrm{ZnO}$ plays an important role in deciding scattering light distribution of lighting device. The $\mathrm{S}-\mathrm{ZnO}$ nanostructure continues to show dominant results in scattering effect comparing to others $\mathrm{ZnO}$ nanostructures. In pc-LEDs configuration with $0.75 \%-2.5 \%$ of $\mathrm{S}-\mathrm{ZnO}$, the light scattering and transmission are the highest among $\mathrm{ZnO}$ nanostructures. Although $\mathrm{ZnO}$ particles are useful in enhancing optical performance, adding $\mathrm{ZnO}$ in the configuration induces the back-scattering effect that damage the lighting efficiency. Any amount of $\mathrm{ZnO}$ will result in light loss from back-scattering, even at $0.25 \% \mathrm{ZnO}$ the value is 0.5 as shown in the figures.

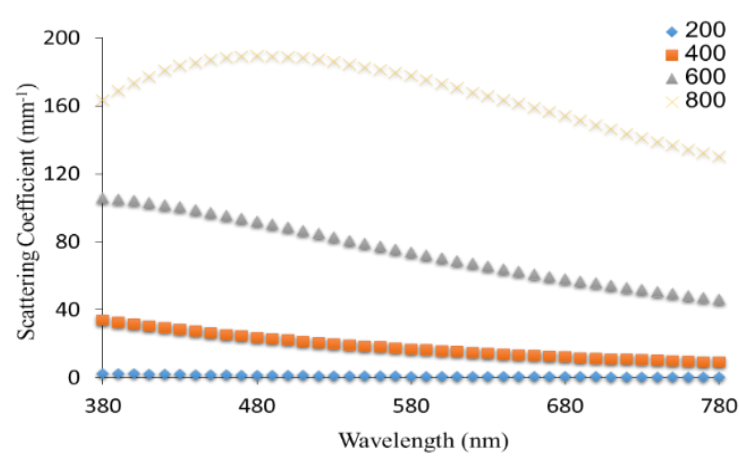

Figure 1. Computation of scattering coefficient of $\mathrm{ZnO}$ particles with various diameters

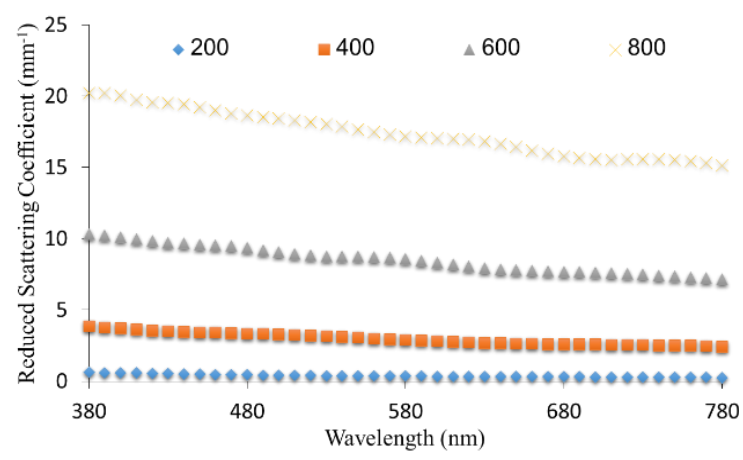

Figure 3. Computation of reduced scattering coefficient of $\mathrm{ZnO}$ particles with various diameters

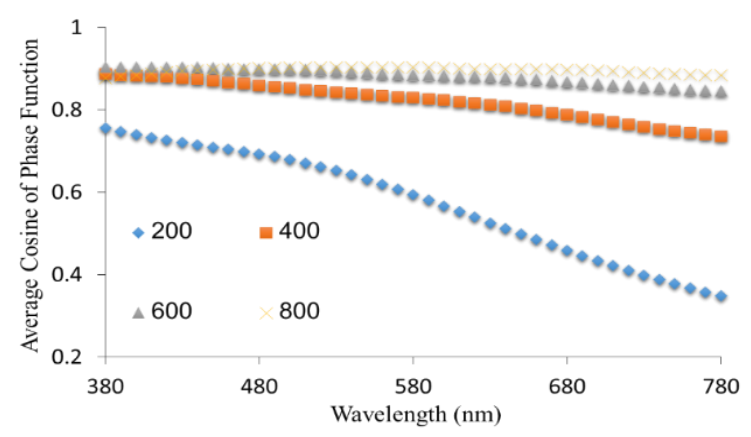

Figure 2. Computation of phase function of $\mathrm{ZnO}$ particles with various diameters

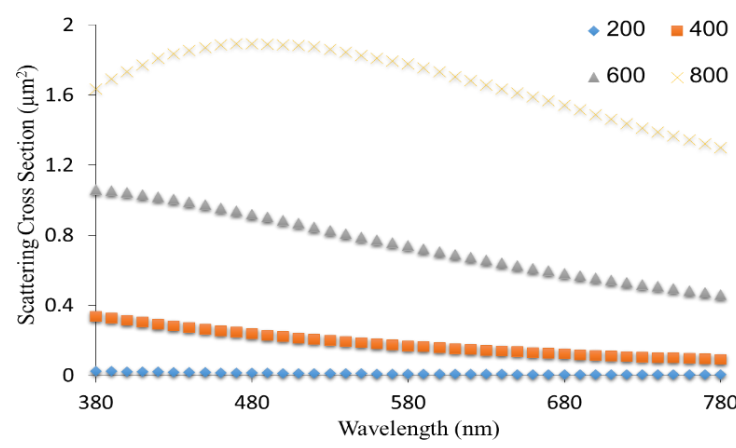

Figure 4. Computation of scattering cross-section of $\mathrm{ZnO}$ particles with various diameters

\section{RESULTS AND DISCUSSION}

In Figures 5(a) and 5(b) are the LEDs device and its parameters in this study, which was built carefully to shows similar to optical-thermal other simulations. LightTools 8.1.0 was employed to replicate the WLEDs with the remote phosphor structure and $\mathrm{ZnO}$ particles. The input data of pc-LEDs are constant that must be determined accurately as shown in Figure 5(c). This physical model of LEDs device has a reflector that is $2.1 \mathrm{~mm}$ in height, $8 \mathrm{~mm}$ in length at its top surface and a $10 \mathrm{~mm}$ bottom as shown in 
Figure 5(d). The remote phosphor layer has a fixed thickness of $0.08 \mathrm{~mm}$ and covers 9 LED chips. The angular CCT distribution of $\mathrm{ZnO}$ pc-LEDs is measured and presented in the graph of Figure 6. According to the figure, color temperature of pc-LEDs improves with the presence of $\mathrm{ZnO}$, in particular, the color quality gradually increase with more $\mathrm{ZnO}$ added in the configuration. This result confirmed that $\mathrm{ZnO}$ is effective in controlling optical properties, more specifically, suitable $\mathrm{ZnO}$ concentration and surface modification can greatly impact the resulted scattering light. Comparing to traditional LEDs device without $\mathrm{ZnO}$, the $\mathrm{ZnO}$ doped configuration the angular-dependent CCT distribution of light emitted from bare LED chip can be viewed as Lambertian type. In Figure 7 are the lumen output calculated from lighting configurations with different $\mathrm{ZnO}$ particles size and concentration. Similar to other optical properties, the light intensity are also affected by addition of $\mathrm{ZnO}$ particles into the configuration. The $\mathrm{ZnO}$ of any type enhance the optical quality of the lighting device. For example, $\mathrm{N}-\mathrm{ZnO}$ nanoparticles improve the light output to the highest value in comparison to the original pc-LEDs without $\mathrm{ZnO}$, which is quite high at $6.32 \mathrm{mcd}$, and other $\mathrm{ZnO}$ doped configurations as a result of excellent light transmission efficiency. In other cases, pc-LEDs devices with $\mathrm{S}-\mathrm{ZnO}$ and $\mathrm{R}-\mathrm{ZnO}$ nanoparticles in the configuration have their color quality enhanced owing to the lighting uniformity. It is confirmed that $\mathrm{ZnO}$ and its structure can be utilized to control pc-LEDs optical quality. However, manufacturer should pay attention to $\mathrm{ZnO}$ concentration as $\mathrm{ZnO}$ doped configuration usually experience light loss resulted from weaker light scattering, especially at high concentration.

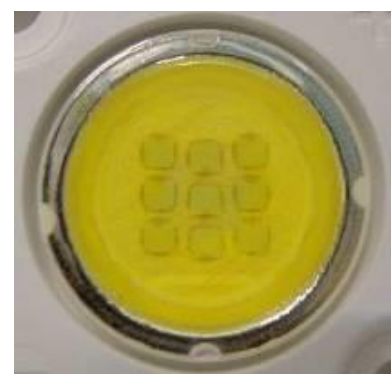

(a)

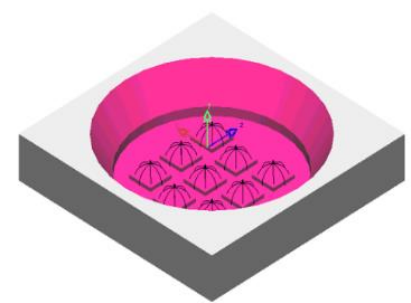

(c)
Lead frame: $4.7 \mathrm{~mm}$ Jentech Size-S

LED chip: $\mathrm{V} 45 \mathrm{H}$

Die attach: Sumitomo 1295SA

Gold Wire: $1.0 \mathrm{mil}$

Phosphor: ITC NYAG4_EL

(b)

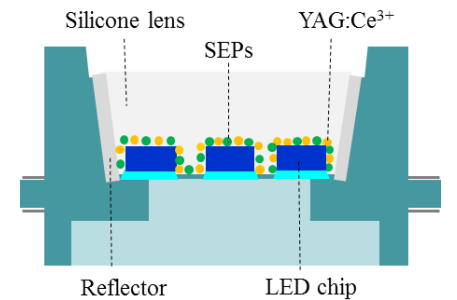

(d)

Figure 5. Photograph of WLEDs structure; (a) Photograph of WLEDs sample, (b) Manufacturing parameter of WLEDs, (c) the simulated WLEDs model, (d) Illustration of 2D WLEDs model

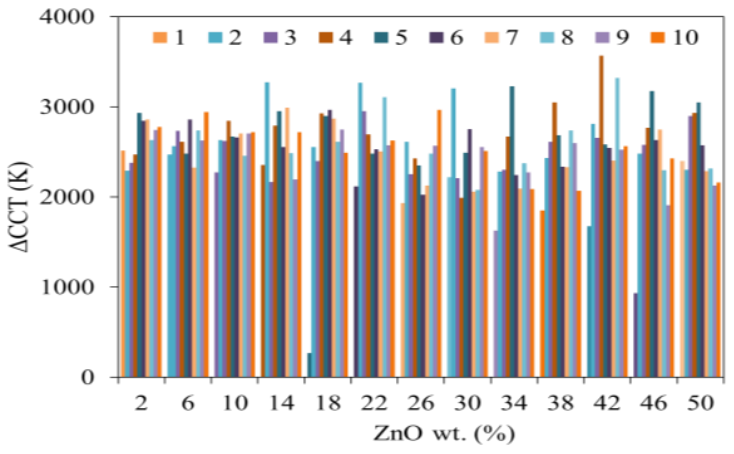

Figure 6. Comparison of CCT deviation of $\mathrm{ZnO}$ particles with various diameters

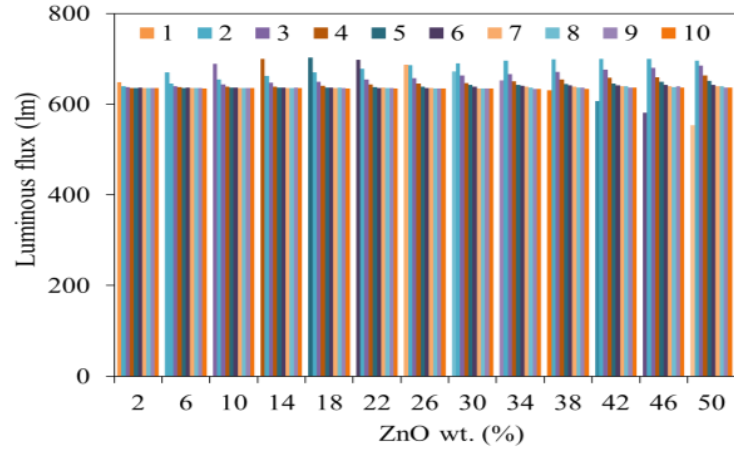

Figure 7. Comparison of luminous flux of $\mathrm{ZnO}$ particles with various diameters 


\section{CONCLUSION}

This research suggest the $\mathrm{ZnO}$ nanoparticles in improving the optical properties of white light. After being integrated into the phosphor layer and placed in the lighting configuration, the enhancement proficiency of $\mathrm{ZnO}$ nanoparticles are confirmed through experiments. The results show that $\mathrm{ZnO}$ benefit the scattering light distribution resulting in higher light quality. The only issue with applying $\mathrm{ZnO}$ particles is that the amount of scattered light is reduced due to scattering traits of $\mathrm{ZnO}$. Through consideration, it is concluded that $\mathrm{N}-\mathrm{ZnO}, \mathrm{S}-\mathrm{ZnO}$, and $\mathrm{R}-\mathrm{ZnO}$ concentrations should be maintained at $0.25 \%, 0.75 \%$ and $0.25 \%$ respectively to minimize the damage on light output while achieve a sufficient standard of color quality. Although $\mathrm{ZnO}$ doped pc-LEDs might not be the optimal enhancement particles, it is still applicable with the benefits it provides while being a low-cost and easy to install. The study of $\mathrm{ZnO}$ effects on lighting properties opens new directions in structure design and shows a method to produce lighting devices with enhanced performance.

\section{ACKNOWLEDGEMENTS}

This research is supported by Industrial University of Ho Chi Minh City (IUH) under grant number 112/HD-DHCN.

\section{REFERENCES}

[1] K. Werfli et al., "Experimental Demonstration of High-Speed 4×4 Imaging Multi-CAP MIMO Visible Light Communications," in Journal of Lightwave Technology, vol. 36, no. 10, pp. 1944-1951, 15 May15, 2018, doi: 10.1109/JLT.2018.2796503.

[2] M. A. Juratli et al., "Noninvasive label-free detection of circulating white and red blood clots in deep vessels with a focused photoacoustic probe," Biomedical optics express, vol. 9, no. 11, pp. 5667-5677, 2018, doi: 10.1364/BOE.9.005667.

[3] Y. Peng et al., "Flexible fabrication of a patterned red phosphor layer on a YAG:Ce3+ phosphor-in-glass for highpower WLEDs," Optical Materials Express, vol. 8, no. 3, pp. 605-614, 2018, doi: 10.1364/OME.8.000605.

[4] Y.-T. Wang et al., "Color conversion efficiency enhancement of colloidal quantum dot through its linkage with synthesized metal nanoparticle on a blue light-emitting diode," Optics letters, vol. 44, no. 23, pp. 5691-5694, 2019 , doi: 10.1364/OL.44.005691.

[5] C. Jaques, E. Pignat, S. Calinon, and M. Liebling, "Temporal super-resolution microscopy using a hue-encoded shutter," Biomedical optics express, vol. 10, no. 9, pp. 4727-4741, 2019, doi: 10.1364/BOE.10.004727.

[6] Y. Shi, S. Ye, J. Yu, H. Liao, J. Liu, and D. Wang, "Simultaneous energy transfer from molecular-like silver nanoclusters to $\mathrm{Sm}^{3+} / \mathrm{Ln}^{3+}, \mathrm{Ln}=\mathrm{Eu}$ or $\mathrm{Tb}$. in glass under UV excitation," Optic Express, vol. 27, no. 26, pp. 38159-38167, 2019, doi: 10.1364/OE.380860.

[7] C. Han et al., "Effect of surface recombination in high performance white-light $\mathrm{CH} 3 \mathrm{NH} 3 \mathrm{PbI} 3$ single crystal photodetectors," Optics Express, vol. 26, no. 20, pp. 26307-26316, 2018, doi: 10.1364/OE.26.026307.

[8] T. C. Nguyen, T. T. T. Can, and W.-S. Choi, "Electrohydrodynamic jet-sprayed quantum dots for solutionprocessed light-emitting-diodes," Optical Materials Express, vol. 8, no. 12, pp. 3738-3747, 2018, doi: 10.1364/OME.8.003738.

[9] C. Huang, Y. Chang, L. Han, F. Chen, S. Li, and J. Hong, "Bandwidth correction of spectral measurement based on Levenberg-Marquardt algorithm with improved Tikhonov regularization," Applied optics, vol. 58, no. 9, pp. 2166-2173, 2019, doi: 10.1364/AO.58.002166.

[10] Y. Xie et al., "Encapsulated room-temperature synthesized $\mathrm{CsPbX} 3$ perovskite quantum dots with high stability and wide color gamut for display," Optical Materials Express, vol. 8, no. 11, pp. 3494-3505, 2018, doi: 10.1364/OME.8.003494.

[11] T. Kozacki, M. Chlipala, and H.-G. Choo, "Fourier rainbow holography," Optics express, vol. 26, no. 19, pp. 25086-25097, 2018, doi: 10.1364/OE.26.025086.

[12] B. Xu, Q. Wu, Y. Bao, C. Gangwei, Y. Wang, and S. Ren, "Time-multiplexed stereoscopic display with a quantum dot-polymer scanning backlight," Applied Optics, vol. 58, no. 16, pp. 4526-4532, 2019, doi: 10.1364/AO.58.004526.

[13] J. Zhang, L. Zhao, X. Bian, and G. Chen, "Ce3 $3^{+} / \mathrm{Mn}^{2+}$-activated $\mathrm{Ca} 7, \mathrm{PO}_{4.2}, \mathrm{SiO}_{4.2}$ : efficient luminescent materials for multifunctional applications," Optics Express, vol. 26, pp. A904-A914, 2018, doi: 10.1364/OE.26.00A904.

[14] S. J.-Boissard, C. Cauwers, and P. Avouac, "CIE 2017 color fidelity index Rf: a better index to predict perceived color difference?," Journal of the Optical Society of America A, vol. 35, no. 4, pp. B202-B213, 2018, doi: 10.1364/JOSAA.35.00B202.

[15] B. G. Assefa, "Realizing freeform lenses using an optics 3D-printer for industrial based tailored irradiance distribution," OSA Continuum, vol. 2, no. 3, pp. 690-702, 2019, doi: 10.1364/OSAC.2.000690.

[16] S. Cincotta, C. He, A. Neild, and J. Armstrong, "High angular resolution visible light positioning using a quadrant photodiode angular diversity aperture receiver, QADA.," Optics Express, vol. 26, no. 7, pp. 9230-9242, 2018, doi: 10.1364/OE.26.009230. 
[17] A. Correia, P. Hanselaer, and Y. Meuret, "Holistic opto-thermal simulation framework for high-brightness light sources based on fluorescent conversion," Optics Express, vol. 27, no. 16, pp. A1324-A1337, 2019, doi: 10.1364/OE.27.0A1324.

[18] W. Wang and L. Cai, "On the development of an effective image acquisition system for diamond quality grading," Applied Optics, vol. 57, no. 33, pp. 9887-9897, 2018, doi: 10.1364/AO.57.009887.

[19] T. P. White, E. Deleporte, and T.-C. Sum, "Feature issue introduction: halide perovskites for optoelectronics," Opt. Mater. Express, vol. 26, no. 2, pp. A153-A156, 2018, doi: 10.1364/OE.26.00A153.

[20] L. Wu et al., "Hybrid warm-white organic light-emitting device based on tandem structure," Optics Express, vol. 26, no. 26, pp. A996-A1006, 2018, doi: 10.1364/OE.26.00A996.

[21] M. C. Aguilar et al., "Automated instrument designed to determine visual photosensitivity thresholds," Biomedical Optics Express, vol. 9, no. 11, pp. 5583-5596, 2018, doi: 10.1364/BOE.9.005583.

[22] C.-Soo Kim, W. Kim, K. Lee, and H. Yoo, "High-speed color three-dimensional measurement based on parallel confocal detection with a focus tunable lens," Optics Express, vol. 27, no. 2, pp. 28466-28479, 2019, doi: 10.1364/OE.27.028466.

[23] A. A. Kaminari, S. Boyatzis, and A. Alexopoulou, "Linking Infrared Spectra of Laboratory Iron Gall Inks Based on Traditional Recipes with their Material Components," Applied Spectroscopy, vol. 72, no. 10, pp. 1511-1527, 2018, doi: 10.1177/0003702818778319.

[24] Q. T. Vinh, P. Bodrogi, and T. Q. Khanh, "Preliminary measure for the characterization of the usefulness of light sources," Optics Express, vol. 26, no. 11, pp. 14538-14551, 2018, doi: 10.1364/OE.26.014538.

[25] C-N. Liu et al., "New scheme of LiDAR-embedded smart laser headlight for autonomous vehicles," Optics Express, vol. 27, no. 20, pp. A1481-A1489, 2019, doi: 10.1364/OE.27.0A1481. 